\title{
Audit of prescription Notes from a Tertiary health centre.
}

\author{
${ }^{1}$ Alagoa P.J., ${ }^{2}$ Mukoro Duke George. ${ }^{3}$ Yeibake Weriwoyingipre Silver \\ ${ }^{1} M B B S, F M C S, F I C S,{ }^{2} . B . s c, M B B S, A T L S_{C E R T}$ DTM\& $H_{\text {Liverpool, }}{ }^{3}$ B.Med.Sci,MBBS \\ ${ }^{1 \& 3}$ Department of Surgery, Niger-Delta University Teaching Hospital. \\ ${ }^{2}$ Department of Accident and Emergency Medicine, Niger-Delta University Teaching Hospital, Bayelsa. Nigeria.
}

\begin{abstract}
:
Introduction: In Medical and pharmaceutical practice, the use and importance of prescription note cannot be undermined. There are reports concerning irrational prescription pattern. The manuscript therefore audits the pattern of prescription writing, strength and deficiencies as well as a review of standards requirement with our prescription templates.

Materials and Method : A total of 740 prescribed medications were reviewed from 225 prescription notes which were collected from the Pharmacy department of a teaching Hospital. Score of 1 and 0 were assigned to presence and absence of parameters respectively,

The results were Tabulated and analyzed with Microsoft excel. Charts were drawn to present our findings.

Results:Deficiencies were observed in every parameter except the name of the patient. Other reported studies observed almost similar occurrences in their Audit.The lowest parameters observed in the study were $2 \%$ for total medication dispensed and 4\% for patient's weight while highest parameters were 100\% for patient's name and $96 \%$ for duration of medication. Average drug medication per prescription note was 3.3.

Conclusion: As a result of these observations as well as audits from other notable reports, a repeated audit and prescription note mentoring is recommended so as to provide quality management of patients, monitor patterns of drugs prescribe and improve the integrity of this medico-legal document.
\end{abstract}

Keywords: Prescription note, audit, pharmaceutical practice,

\section{Introduction}

Prescriptions have been in use since ancient times for the use of the management of patients. The insignia $\mathrm{RX}$ is used.The people permitted to write a prescription are high level practitioners such as Physicians, Veterinarians, Dentists, Podiatristsand Mid-level practitioners such nurse as practitioners, physician assistants, optometrists, pharmacists. Prescription writing is to guarantee patients safety ${ }^{1}$, However, current practices in writing such an this important note have been questionable ${ }^{2}$ and therefore ,necessitates the need for regular self,unit or pharmaceutical audits ${ }^{3 \& 4}$.On this background, a cross-sectional survey was carried-out on past prescription notes stored within the pharmacy of a Teaching Hospital. Local audits have not yet been published. The consumables such as Foley's catheter, urine bag or syringes, written within these notes were exempted from the study.Only drug prescriptions were reviewed retrospectively. Ethical clearance was obtained from the Research and Ethical committee of the Teaching hospital.

\section{Materials and Method.}

A month was balloted out of 12 months of the year.A 6 days consecutive series of prescription notes of the random selected month were identified from the storage of past prescription notes stored in the pharmaceutical department of Niger-Delta University Teaching Hospital, which is located in the Southern part of Nigeria.

The prescription of 6 consecutive days, making-up a total 225 prescription notes, was observed for the following parameters:the use appropriate generic name or trade name of the drug, the strength of the composition of the drug, number of tablet/drops of the drugs, timing(direction) for the drug, total doses to be dispensed. Identifications and authentication for a prescription was examined such as the prescriber's name and signature, patients name, age,weight as well as legibility and cancellation of open spaces in the written prescription note.A score of one was assigned if the assessed parameter was present or observed,while a score of 0 was assigned when absent. The parameters were tabulated and scores calculated while analysis was done using Microsoft Excess software.Ethical clearance was obtained from the Research Ethical committee of the Niger-Delta University Teaching Hospital.

\section{Result:}

A total of 740 drugs were prescribed in 225 prescription notes.Average numbers of drug prescription was 3.3 drugs. Results revealed that generic name of drugs which is the most appropriate for prescription and most internationally accepted were used for $53 \%$ of the prescribed drugs .See Figure1 


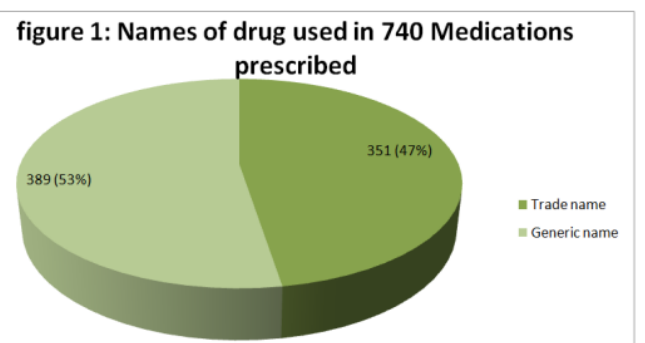

Figure 2:Bar charts of parameters in a prescription sheet

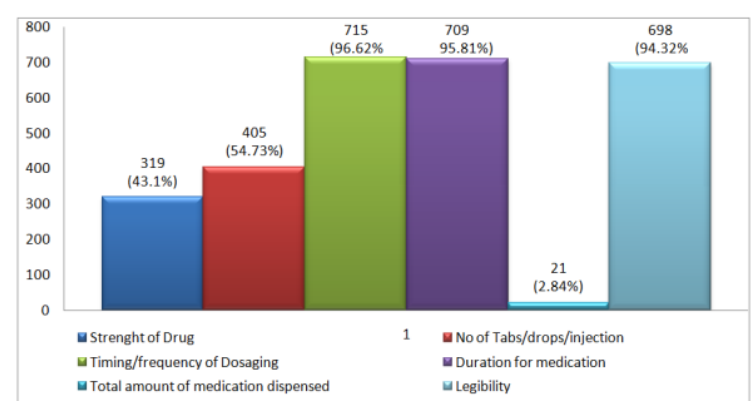

Total amount of dosage prescribed and weight of the patients were poorly indicated in prescription note, results were at rate of $2.84 \%$ and $4.44 \%$ respectively ( see figure 2 and figure 3 ). Best among the assessed parameters for an ideal prescription was patient's name towhom the drug prescription belonged. Most of parameters where above 75 percent with exception to the drug strength, the number of tablets or droplets to be taken, the total numbers of medication dispensed,patient sex, weight and age of the patient.

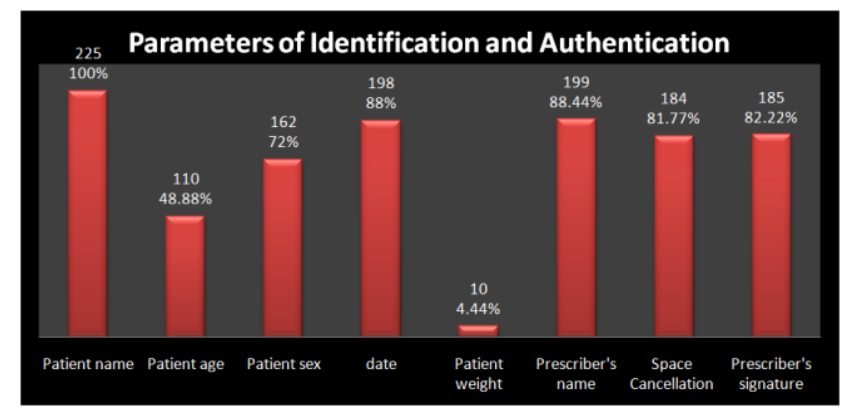

IV. Discussion:

The prescription note is among the most commonly used tools in the care of patients both as out-patient and in-patient. It the most important tool for communication between the physician and the pharmacist concerning patients care. It is also a medium through which patients may be informed about their care.Despite its importance, caution to ensure the legibility, accuracy and completeness towards the content of this vital note had been thrown to the wind.The first group of parameters that must be in a prescription note is the Patient'sIdentification parameters ${ }^{\text {figure } 3}$. The patients name prevents wrong prescription and administrations. There is ample international evidence that poor quality prescription writing increases the risk of serious medication errors ${ }^{5}$. Our studies showed that patients' names were in all prescriptionsalthoughthere were some without age, sex, or weight (see figure 3).The observed rates were 100, 48, 72 and 4 percent respectively, in comparism with another ${ }^{6}$ study which stated $98.72,48.60,46.55$, and $2.03 \%$ for patient name, age sex and weight respectively. Incomplete notes are not peculiar to our situation only. The age is very vital to guide for dispensersof medication so as to ensure that appropriate dosage are written therein. Even when dosage appears appropriate for age, there is yet need to write the weight especially for age less than 12 years. Therefore, In areas where malnutrition make up large number of paediatric cases seen in an health center or health post, the weight of children must be written to prevent over or under-dosaging. The prescription note reflects the physician's skill in the diagnosis and attitude towards selecting the most appropriate cost effective treatment thru list of the drugs written in the note ${ }^{1 \& 7}$. The drug name is the key component of a prescription note ${ }^{\text {Figure }{ }^{1}}$. The international approved drug name(Generic) must be written clearly and legibly, if possible in capital letters.43\% had the dose strength of the drug written and 55\% 
of numbers of tabs or drops mentioned and 96 percent for the frequency/timing in contrast to a study ${ }^{6}$ which reported $52.67 \%$ ) prescriptions were having doses mentioned.

The use of generic drug names was better $(73.53 \%)$ in a similar audit ${ }^{6}$ while ours revealed $53 \%$. The rate of prescriber's signature in the aforementioned study ${ }^{6}$ was similar to ours see figure3. Trade(Brand) names are not allowed in writing a medication because there are so many names in use nowadays. The trade names can sway dispensers who may not ask for second opinion before giving- out wrong $\overline{\text { drugs }}$ to another client based on their personal understanding and little knowledge. The chances of such mistakes are also so high where large number of clients are to be seen.Other areas where mistakes can be made is when chemical description or abbreviations are used such as $\mathrm{FeSO}_{4}$ or $\mathrm{ZNSO}_{4}$. Exception to writing brand names may include drug such as Aminophylline, Ciclosporin, Diltiazem long acting formulations, Lithium, Nifedipine Theophylline and so on. In such instance, both names must be written alongside each other example: Ampiclox (Becham) or in combination product. The use of generic name of a product was 53 percent from our study. There is dire need to improve acquaintance with the generic name of commonly prescribed drugs by physicians and the increase in awareness amongst physicians and consumer's about the concept of essential medicines, advantages of generic drugs prescription \& use of rational combinations ${ }^{8}$.

Most drugs have formulations and/or strengths of a preparation. It is important that details are correctly stated on the prescription and the mode of consumption of such drug, example oral, inhaler, or injection. This would ensure that patient receives correct products. The dosage of a drug was written only in 43 percent of 740 medications. Reasons may be that physicians have replaced dosage with number of tablets with abbreviationsuch asT or have forgotten the dosage of the drug. The reluctance to check-up drug formulary is another factor to absence dose on prescription. Decimal place are not allowed in drug prescription. Examples of correct dose include $3 \mathrm{mg}$ or $0.5 \mathrm{mls}$ and (not $0.1 \mathrm{mg}$ or $.5 \mathrm{ml}$ ). Micrograms, nanograms and units must be written in full and not abbreviated. Internationally recognized abbreviation such as $\mathrm{g}$ (grams), ml(milliliters), and $\mathrm{mg}$ (milligrams) are acceptable.The concentration of liquid preparations should always be written by the physician. If not written,the pharmacist should ensure it is written beside the drug prescribed, and dosage calculated correctly before dispensing such drugs. Route of administration must be written with acceptable abbreviation such as IV,SC,NG,JEJ,PR,TOP,NEB,LE,PO,IM,PEG,S/L, and PV.The date of a prescribed medication must be stated.The frequency of a drug should be stated with appropriate abbreviations, the following Latin abbreviations are arceptable:o.d. for daily ; o.m. or mane as in the morning, o.n. or nocteat night ,t.d.s for three times daily, stat for immediately, p.r.n. for (when required).Frequency of drug was stated in 96.62 percent of the written notes. The authentication and authority of a prescription depends on prescriber's name and signature which rates were 91 and 84 percent respectively as observed from the study.Spaces left in a prescription note should be crossed-off to prevent other medication written into a note without physician notice .A written note may be considered as the physician attitude, knowledge level towards a particular disease, therefore, health practitioners must be knowledgeable enough and thorough in what he or she writes in a prescription note ${ }^{9}$. Other parameters which were not in the studied note template has been observed to be quite useful information for patient management and drug control include boxes indicating drug allergies, confirmation boxes for indicating appropriateness of prescription as well as prescriber's telephone number for contact. Our studied template however has a place for pharmacy name and signature. Our template in this study confirms a need to update by including other information as well. Similar needs was also felt by another studies as well ${ }^{10}$. Finally, it would not be out of place to say that patient's and prescribers identifications as well as drug prescribed has legal implications in our world where court cases for malpractice is on the rise. The limitations observed in this study necessitate the need for repeated audit which has been known to improve quality of drug prescription notes. ${ }^{1 \& 4}$

\section{Conclusion:}

Current practices in writing prescription notes have been noticed to be in-appropriately written and obscured with deficiencies, therefore, these calls for regular auditing, mentoring and maintaining of standards of this vital medico-legal document.

\section{References}

[1] Teichman PG, CaffeeAE :Prescription writing to maximize patient safety; Family Practice Management 9 (7): 27-30 (2002)

[2] Arin Bhattacharya, AnuragSatpathy, PrashantTiwari: Assessment of Prescription Pattern of Toothache and tooth extraction Patient's Prescription: A Prescription Survey Study from Various Hospitals and Clinics of Bhubaneswar and Cuttack, Pharmacologyonline 3: 135-148 (2011).

[3] NareshJyoti and SharanjitKaur: To analyze the Impact of Serial Prescription Audits with Active Feedback on Quality of Prescription Behaviour.JClinDiagn Res. 2013 April; 7(4): 680-683.

[4] Mathur M, Dandiya PC. Indian J Pharmacol.Prescribing pattern for outpatients in government hospitals in Jaipur2004;36:383-4.

[5] Joshi MC. Medication error reporting through prescription auditing. Express Healthcare Management. 2005;June Issue dated 1630June 2005

[6] Chandrashekar K1, Saritha MK2, VinayakMeti : Prescription audit in tertiary care centre ,Short Communication; Asian J Med CliSci ,May -Aug 2012 ,Vol-1, Issue- 2. 
[7] Srishyla MV, Krishnamurthy M, Nagarani MA, Clare SM, Andrade C, Venkataraman BV. Prescription audit in an Indian hospital setting using the DDD (Defined Daily Dose) concept. Indian J Pharmacol. 1994;26:23-28.

[8] Arin Bhattacharya, AnuragSatpathy, PrashantTiwari: Assessment of Prescription Pattern of Toothache and tooth extraction Patient's Prescription: A Prescription Survey Study from Various Hospitals and Clinics of Bhubaneswar and Cuttack,Pharmacologyonline 3: 135-148 (2011).

[9] Tripathy K.D, Text book of Medical Pharmacology. $6^{\text {th }}$ edition,Jaypee brothers medical publishers (P) Ltd; p.167.

[10] Arin Bhattacharya, AnuragSatpathy, PrashantTiwari: Assessment of Prescription Pattern of Toothache and tooth extraction Patient's Prescription: A Prescription Survey Study from Various Hospitals and Clinics of Bhubaneswar and Cuttack,Pharmacologyonline 3: 135-148 (2011). 